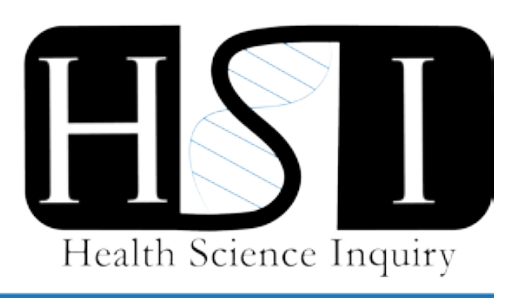

\title{
Using precision medicine to reduce falls in individuals with Alzheimer's disease
}

\author{
Shirin Modarresi ${ }^{1}$, Maryam Ghodrati ${ }^{1}$, Erfan Aref-Eshghi ${ }^{2}$ \\ ${ }^{1}$ Department of Health and Rehabilitation, University of Western Ontario; ${ }^{2}$ Department of Pathology, University of \\ Western Ontario
}

Falls are considered a significant public health problem in the older adult population ( $>65$ years) [1]. Consequences of falls can be major injuries such as hip fractures or head traumas, hospitalization and even death [1]. Aside from these more seemingly debilitating outcomes, falls can also lead to adverse psychological effects such as depression and loss of independence [2]. The economic impact of falls is also substantial; in Canada alone the associated direct costs of falls is more than two billion CAD annually [3]. The problem of falls and their consequences is more severe in individuals with Alzheimer's disease (AD), as they fall at least twice as often as cognitively healthy older adults, incur more severe injuries and experience lower recovery rates [4]. Currently, there are approximately 44 million people with $\mathrm{AD}$ or other forms of dementia, and this number is expected to triple in the next 40 years [5]. Despite the many studies conducted in an effort to reduce falls, we are still facing their negative impacts. A systematic review of fall prevention programs (e.g. physiotherapy, strength training, medication review) in people with dementia demonstrated that these programs were beneficial for reducing falls only in some of the participants [6]. It is essential to discover a successful fall prevention method for people with $A D$, as it would lead to a better quality of life for the patients and a lower economic burden for society. Bearing in mind that current fall prevention strategies are not effective for all individuals with dementia, it is time to investigate this matter from a novel perspective.

$A D$ is an etiologically heterogeneous condition caused by different genetic and environmental factors in different individuals [7]. The rate at which people with $A D$ lose their cognitive and physical abilities is determined by the originating factors as well as the constitutional genetic makeup of the patients [8]. This indicates that not all people with $A D$ fall due to a shared etiology or possess the same risk factors for falls [9]. To a great extent, the numerous factors that contribute to falls in people with $A D$, including severity of cognitive impairment, vision problems, balance and gait impairment and disease co-morbidities, are influenced by the patient's genetic background. Clearly, each patient requires a different approach to the prevention of falls, as the etiology and risk factors for falls vary in different patients. In addition, just by treating the noticeable risk factors (e.g. vision impairment) in a person with $A D$, the problem of falls cannot be eliminated, as there could be other unknown contributing factors. Since the risk factors can potentially vary among individuals with AD that fall, the genetic make-up or the more in-depth underlying reason for this difference amongst these individuals can be strikingly different as well [9]. In this article we propose that approaches used in precision medicine to determine who should receive which treatment can be highly beneficial to this line of investigation. Besides the patient's original genetic makeup (the genome), which influences the entire progression of the disease without changing during a patient's lifetime, other layers of gene expression regulation (i.e. epigenomics, transcriptomics, proteomics and metabolomics) are highly plastic and can immediately reflect any change in the status of any primary, secondary, or interactive factors that determine the risk of falls in people with AD.8 The improvement and reduction in cost of genomic technologies in recent years have made feasible the exploration of millions of such biomarkers in relation to response to various fall prevention programs. With the improvement in sensitivity and throughput of such tools, we are now able to perform non-invasive liquid biopsies from easily accessible tissues (e.g. peripheral blood plasma, urine and saliva) to search for biomarkers that reflect the biological processes occurring in the more relevant non-accessible organs, e.g. the brain in AD. The advent of highly computational methodologies such as data mining and machine learning has removed the obstacles in interpretability and applicability of the discovered biomarkers in the age of multi-omics [10].

In conclusion, it is imperative to reiterate that the number of individuals with $A D$ is increasing, and fall-related injuries, which are one of the most expensive medical conditions associated with the disease, are also at an incline. While many risk factors for falls have been identified, and they can be used to determine who is most 
likely at a higher risk for falling, we lack fall prevention strategies that can be used for every patient with AD. In this commentary, we propose methods used in precision medicine to stratify patients based on their genomic and epigenomic profile and direct appropriate interventions to the specific deficits. Unlike other medical fields, precision medicine using genetics technology has not been used in rehabilitation sciences. This approach can potentially lead to saving time and reducing healthcare costs associated with falls and will also open doors for further investigations in other conditions in the same field.

\section{References}

[1] Tinetti, M.E., Speechley, M., Ginter, S.F. (1988) Risk factors for falls among elderly persons living in the community. N Engl J Med., 319 (26), 1701-1707.

[2] Deshpande, N., Metter, E.J., Lauretani, F., Bandinelli, S., Guralnik, J., Ferrucci, L. (2008) Activity restriction induced by fear of falling and objective and subjective measures of physical function: a prospective cohort study. J Am Geriatr Soc., 56 (4), 615-620.

[3] Stinchcombe, A., Kuran, N., Powell, S. (2014) Report summary-seniors' falls in canada: Second report: key highlights. Chronic Dis Inj Can., 34, 2-3.

[4] Morris, J.C., Rubin, E.H., Morris, E.J., Mandel, S.A. (1987) Senile dementia of the Alzheimer's type: an important risk factor for serious falls. J Gerontol. 42 (4), 412-417.

[5] Manuel, D.G., Garner, R., Finès, P., Bancej, C., Flanagan, W., Tu, K., Reimer, K., Chambers,
L.W., Bernier, J. (2016) Alzheimers and other dementias in Canada, 2011 to 2031: a microsimulation Population Health Modeling (POHEM) study of projected prevalence, health burden, health services, and caregiving use. Popul Health Metr., 14 (1), 37.

[6] Oliver, D., Connelly, J.B., Victor, C.R., Shaw, F.E., Whitehead, A., Genc, Y., Vanoli, A., Martin, F.C., Gosney, M.A. (2007) Strategies to prevent falls and fractures in hospitals and care homes and effect of cognitive impairment: systematic review and metaanalyses. BMJ, 334 (7584), 82.

[7] Schellenberg, G.D. (1995) Genetic dissection of Alzheimer disease, a heterogeneous disorder. Proc Natl Acad Sci U S A, 92 (19), 8552-8559.

[8] Cacabelos, R., Fernández-Novoa, L., Lombardi, V., Kubota, Y., Takeda, M. (2005) Molecular genetics of Alzheimer's disease and aging. Methods Find Exp Clin Pharmacol., 27, 1-573.

[9] Mayeux, R., Ottman, R., Tang, M.X., NoboaBauza, L., Marder, K., Gurland, B., Stern, Y. (1993) Genetic susceptibility and head injury as risk factors for Alzheimer's disease among community-dwelling elderly persons and their firstdegree relatives. Ann Neurol., 33 (5), 494-501.

[10] Aref-Eshghi, E., Rodenhiser, D.I., Schenkel, L.C., Lin, H., Skinner, C., Ainsworth, P., Paré, G., Hood, R.L., Bulman, D.E., Kernohan, K.D., Boycott, K.M. (2018) Genomic DNA Methylation Signatures Enable Concurrent Diagnosis and Clinical Genetic Variant Classification in Neurodevelopmental Syndromes. Am J Hum Genet., 102 (1), 156-174. 


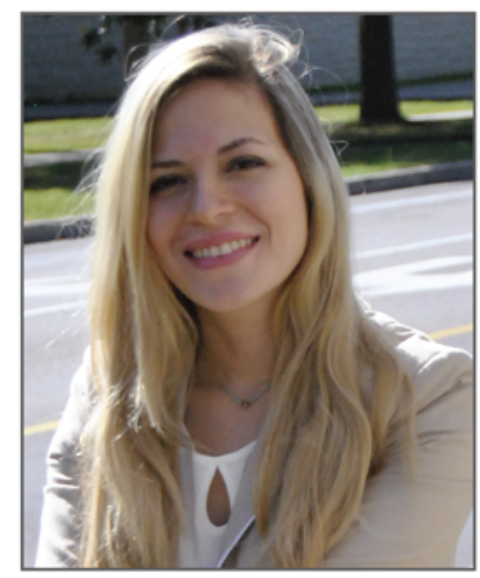

Shirin Modarresi is a PhD candidate in the combined master of Physical Therapy-PhD program in the Health and Rehabilitation department at Western University. She completed her MSc in Medicine (neuroscience stream) at Memorial University of Newfoundland. She obtained her BSC (honors) in biology and psychology from the University of Toronto. The focus of her $\mathrm{PhD}$ research is finding novel rehabilitation methods and strategies for people with chronic neurological disorders, specifically Alzheimer's disease and chronic regional pain syndrome.

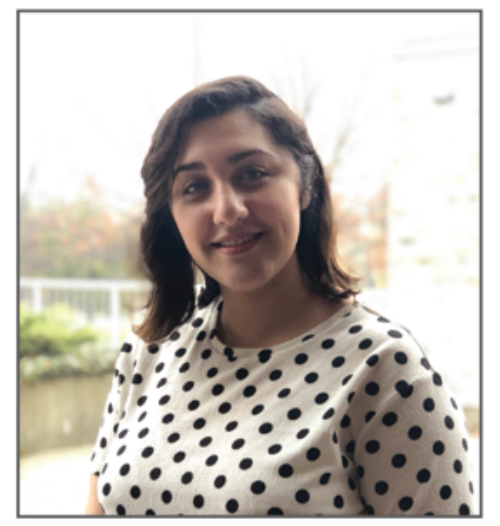

Maryam completed her Bachelor and Master degrees with high distinction in Physical Therapy and worked as a physiotherapist for about two years before attending Western University to pursue her PhD in Physical Therapy. Her interest in chronic musculoskeletal pain has inspired her current research focusing on proposing a novel approach to simultaneously evaluating both gender and biological variables in the same model to better explain the direct and interactional effects on pain, distress, disability, and subsequent recovery with the supervision of Dr. David Walton.

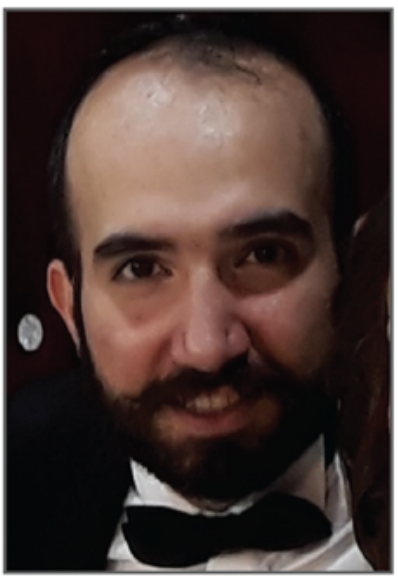

Erfan is a trained medical practitioner with a PhD in Human Genetics. He has an interdisciplinary research background ranging from molecular genetics to statistics, clinical epidemiology, and bioinformatics. He is currently a postdoctoral research associate at the university of Western Ontario. He applies computational modelling and machine learning to genomic and epigenomic data to design methods that improve the molecular diagnostics of a variety of constitutional hereditary disorders as well as cancer. 